\title{
Studies on the Nephrotoxicity of Aminoglycoside Antibiotics and Protection from These Effects (8): Protective Effect of Pyridoxal-5'-Phosphate against Tobramycin Nephrotoxicity
}

\author{
Ryoji KOJIMA, Mikio ITO and Yoshio SUZUKI \\ Department of Pharmacology. Faculty of Pharmacy. Meijo University, \\ 15 Yagoto Urayama, Tenpaku-ku. Nagoya 468. Japan \\ Accepted September 13, 1989
}

\begin{abstract}
We investigated the effect of pyridoxal-5'-phosphate (PALP) on tobramycin (TOB)-induced nephrotoxicity in rats. Paper electrophoretic analysis showed that in the mixture of TOB and PALP, the spot corresponding to TOB alone almost disappeared and the spot associated with TOB overlapped with that associated with PALP, although the spots of TOB alone and PALP alone were observed as single spots on the cathode and anode sides, respectively. The overlapping of both compounds indicated that TOB could directly interact with PALP in vitro. In the assay of TOB binding to renal brush border membranes (BBMs). PALP significantly inhibited the binding of TOB to BBMs by interacting with TOB outside of BBMs vesicles. Intrarenal TOB levels in rats receiving TOB and PALP were lower than those in rats given TOB alone. Combination with PALP markedly suppressed the urinary protein content, urinary $N$-acetyl- $\beta$-Dglucosaminidase activity and blood urea nitrogen content elevated by TOB, and also reduced the degree of TOB-induced renal tubular cell necrosis. These results indicate that PALP protects the rat kidneys from TOB-induced nephrotoxicity and that the protective effect of PALP may be due to the reduced intrarenal TOB concentration and less binding of TOB to BBMs induced by the interaction of PALP with TOB.
\end{abstract}

We have already reported that latamoxef (LMOX), an oxacephem antibiotic, prevents tobramycin (TOB)-induced nephrotoxicity in rats and that the protective effect of LMOX is partly due to the ability of LMOX to reduce the intrarenal TOB concentration (1). We have found that the inhibitory effect of LMOX on intrarenal TOB level resulted from the inhibition of the binding of TOB to renal brush border membranes (BBMs) (2) by a molecular interaction between TOB and LMOX (3).

From our previous study using LMOX, it was hypothesized that a compound which has a negative charge in its molecule and is excreted into the urine might prevent TOBinduced nephrotoxicity by reducing the intrarenal TOB level and by inhibiting the binding of $T O B$ to BBMs through a molecular interaction between TOB and the compound.

As shown in Fig. 1, pyridoxal-5' -phosphate
(PALP), an active form of vitamin $B_{6}$, has a phosphate group in its molecule and is negatively charged at physiological pH. PALP also has an aldehyde group, which can form a Schiff base by binding covalently to amino groups of TOB. In general, excess amourits of vitamins are excreted into the urine without converting them into metabolites, when large amounts of vitamins are administered. Therefore, in the present study, to examine the ability of PALP to prevent TOB-induced nephrotoxicity, we designed experiments to monitor the following: 1) in vitro interaction of TOB with PALP, 2) the effect of PALP on TOB binding to BBMs, 3 ) the effect of PALP on intrarenal TOB concentration, and 4) the effect of PALP on the nephrotoxicity of TOB in vivo.

In addition, in order to obtain some information about the mechanism of the action 


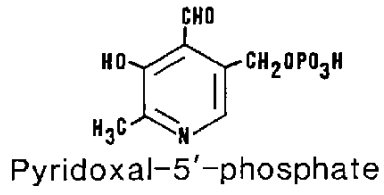<smiles>Cc1nc(N(Cl)Cl)cc(CO)c1O</smiles>

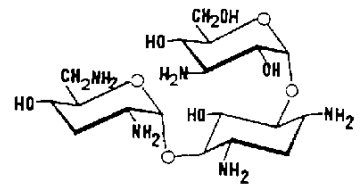<smiles>Cc1ncc(CO)c(CO)c1O</smiles>

Pyridoxine

Tobramycin<smiles>Cc1cc(C(=O)O)c(CO)cn1</smiles>

4-pyridoxic acid

Fig. 1. Chemical structures of tobramycin, pyridoxal-5'-phosphate, pyridoxal, pyridoxine and 4pyridoxic acid.

of PALP on the interaction with TOB and the TOB binding to BBMs, pyridoxal (PL) and pyridoxine ( $P N)$, the precursor of PALP, and 4-pyridoxic acid (4-PA), a metabolite of PALP, were examined.

\section{Materials and Methods}

Drugs: TOB was provided by Shionogi Co., Ltd. (Osaka, Japan). PALP, PL and PN were purchased from Tokyo Kasei (Tokyo. Japan). and 4-PA was obtained from Sigma Chemicals (St. Louis, MO, U.S.A.).

Animals: Male Sprague-Dawley rats weighing $230 \mathrm{~g}$ (Shizuoka Laboratory Animal Center, Shizuoka, Japan) were used in a!l experiments. These animals were housed in an air-conditioned room at $24 \pm 1{ }^{\circ} \mathrm{C}$.

Paper electrophoretic analysis: According to our previously reported method (3), paper electrophoresis analysis was used to clarify whether PALP interacts with TOB in vitro. Briefly. TOB alone. PALP alone and a mixture of both drugs (molar ratio, TOB:LMOX $=1: 2$ ) were dissolved in $\mathrm{H}_{2} \mathrm{O}$, and the $\mathrm{pH}$ was adjusted to 7.4 by the addition of $\mathrm{HCl}$ or $\mathrm{NaOH}$. Each solution containing drugs was incubated for 10,30 and $60 \mathrm{~min}$ at $37^{\circ} \mathrm{C}$, and aliquots sampled at a suitable time were subjected to paper electrophoresis. The svots associated with TOB and PALP on the paper were visualized by the ninhydrin reaction and ultraviolet irradiation $(\lambda=254 \mathrm{~nm})$, respectively. In addition, the mixtures of TOB and PL, PN or 4-PA were also analvzed.

Assay of TOB binding to BBMs: The assay of TOB binding to BBMs, which were isolated from rat kidney cortex by the method of $\mathrm{Ca}$ precipitation $(4,5)$, was carried out using the method of Ishikawa et al. (6). In brief, in a general assay, $200 \mu l$ of membrane suspension (200-250 $\mathrm{kg}$ protein) was incubated at $37^{\circ} \mathrm{C}$ for $10 \mathrm{~min}$ with $200 \mu \mathrm{l}$ of $0.2 \mathrm{mM}$ of TOB in $20 \mathrm{mM}$ HEPES/Tris buffer ( $\mathrm{pH} \mathrm{7.4)} \mathrm{con-}$ taining $100 \mathrm{mM}$ mannitol, followed by centrifugation at $45.000 \times \mathrm{g}$ for $30 \mathrm{~min}$ at $4^{\circ} \mathrm{C}$. After removal of the supernatant, $200 \mu \mathrm{l}$ of $\mathrm{H}_{2} \mathrm{O}$ and $5 \%$ trichloroacetic acid were added. centrifuged at $1,600 \times \mathrm{g}$ for $20 \mathrm{~min}$, and the resultant supernatant was used for measuring TOB concentration. The TOB concentration was determined by means of substratelabeled fluorescent immunoassay (SLFIA, Ames TDA tobramycin assay kit, MilesSankyo Co. Ltd., Toyo, Japan) (7). The final concentrations of $0.2 \mathrm{mM}$ of TOB and $0.4,0.8$, 2 or $4 \mathrm{mM}$ of PALP were used for the binding assay. According to the binding assay described above, we designed experiments to determine the following: 1) effect of simultaneous treatment and pretreatment with PALP on TOB binding to BBMs: 2) effect of the reaction mixture of $T O B$ and $P A L P$, which 
was preincubated for $3 \mathrm{hr}$ at $37^{\circ} \mathrm{C}$ to make a complex of both compounds, on TOB binding to BBMs; and 3) the binding of TOB to PALPtreated and non-treated BBMs. The effects of $P L, P N$ and 4-PA on TOB binding to BBMs also were examined using the same concentrations as those of PALP.

Measurement of the intrarenal TOB concentration: Drug treatments were designed as follows: 1) Rats were given TOB (90 mg/kg/ day, s.c.) alone or TOB and PAL.P (200 mg/ $\mathrm{kg}$, p.o. $1 \mathrm{hr}$ before TOB injection) for 3 days: 2) $\mathrm{TOB}(70-150 \mathrm{mg} / \mathrm{kg}$, s.c.) alone or TOB and PALP (156-333 $\mathrm{mg} / \mathrm{kg}$, p.o. $1 \mathrm{hr}$ before TOB injection) were administered to rats; and 3) Treatments with PALP (200 mg/kg. p.o.) to rats were performed 5 and $1 \mathrm{hr}$ before and after single 5.c. injection of TOB as well as simultaneously with TOB treatments. Twentyfour hours after the drug administration, the left kidneys were removed and the intrarenal TOB concentration was determined by the previously reported method (1). except for centrifugation at $9.000 \times \mathrm{g}$ for $15 \mathrm{~min}$ and the measurement of TOB concentration by SLFIA.

In vivo nephrotoxicity studies: 1) Drug treatment: Animals were given daily s.c. doses of TOB $(90 \mathrm{mg} / \mathrm{kg})$ alone or TOB and p.o. doses of PALP (200 or $510 \mathrm{mg} / \mathrm{kg}$ ) for 15 consecutive days. Daily p.o. administrations of PALP were carried out 1 hr before the TOB injection.

2) Measurements of urinary protein content and $N$-acetyl- $\beta$-D-glucosaminidase (NAG) activity, and blood urea nitrogen (BUN) content: After the beginning of drug treatment, the twenty-four hour urine was collected at suitable intervals, and blood was obtained from the jugular vein on the 15th day. Urinary protein content and NAG activity. as well as BUN content, were measured as in our previous report (1). In addition to the measurements of biochemical parameters in the urine and blood, the histological observation of kidneys also was performed on the sections stained with Periodic Acid-Schiff (PAS) for light microscopy, as described previously (1)

Statistical analysis: The data were subjected to one-way analysis of variance and the Duncan multiple range test or non-parametric statistics. When only two groups were used. the data were analyzed by Student's $t$-test. The data in the text and figures are expressed as the meants.D. Differences were considered significant if the $P$ value was $<0.05$.

\section{Results}

\section{Analysis of interaction of TOB and PALP by paper electrophoresis}

As shown in Fig. 2A, spots of TOB and PALP were observed as single spots on the cathode and anode side, respectively. When the solutions containing TOB and PALP were incubated at $37^{\circ} \mathrm{C}$ for various time. the spot corresponding to TOB alone almost disappeared, and the spot associated with TOB overlapped with that associated with PALP from the cathode to anode side. The degree of the overlap seemed to become greater as incubation time increased. The increase in the molar ratio of PALP to TOB accelerated the degree of the interaction between both compounds (data not shown).

Additionally. when the mixture of TOB and $P L$ was analyzed by paper electrophoresis, the spot associated with TOB overlapped with that associated with $P L$ on the cathode side. indicating that $P L$ also directly interacted with $\mathrm{TOB}$. However, the degree of the interaction between these compounds seemed to be less than that observed between TOB and PALP. In the mixture of TOB and PN, only an alteration of the mobility of PN on the paper was detected, and 4-PA showed no interaction with TOB (Fig. 2B).

\section{Assay of TOB binding to BBMs}

As shown in Fig. 3A, the simultaneous addition of TOB $(0.2 \mathrm{mM})$ and PALP $(0.4$ to 4 $\mathrm{mM})$ to the BBMs fraction significantly inhibited TOB binding to BBMs by about 20 to $90 \%$. The addition of the reaction mixture of TOB and PALP $(0.8$ to $4 \mathrm{mM})$, which was preincubated for $3 \mathrm{hr}$ at $37^{\circ} \mathrm{C}$, resulted in less binding of TOB to BBMs than that observed in the case of simultaneous addition of both drugs (Fig. 3B). Pretreatment of BBMs with PALP, except in the case of an $0.8 \mathrm{mM}$ dose of PALP, did not result in less binding of TOB to BBMs, as compared with the results from the simultaneous addition of both compounds. It was of interest that the pretreatment with 0.8 $\mathrm{mM}$ of PALP resulted in increased TOB binding to BBMs. (Fig. 3C) 
A

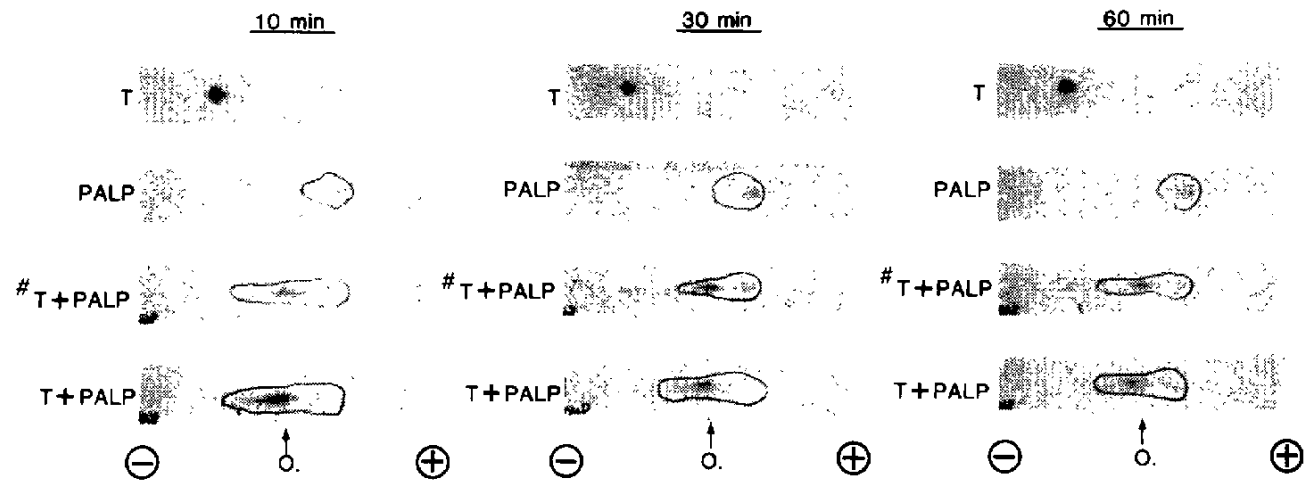

B
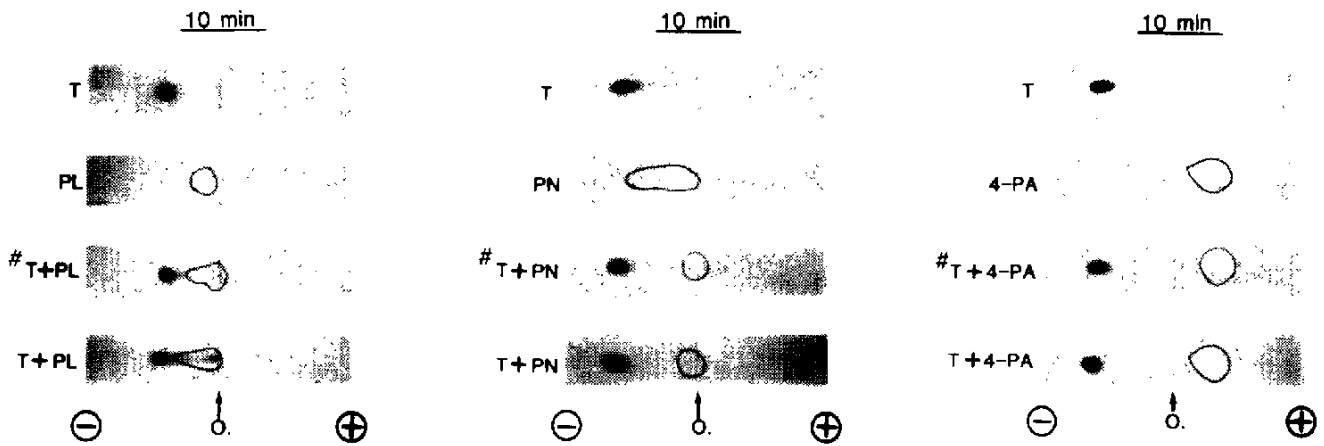

Fig. 2. Analysis of the interaction of TOB and PALP. PL. PN or 4-PA by paper electrophoresis. Black spots and circled areas on the paper indicate the spots corresponding to TOB and PALP. PL, PN or 4-PA, respectively. Each mixture of TOB and other compounds (molar ratio. TOB:compound $=1: 2$ ) was incubated for 10,30 and $60 \mathrm{~min}$ at $37^{\circ} \mathrm{C}$. At a suitable time, aliquots were subjected to paper $(2.5 \times 15$ $\mathrm{cm}$ ) electrophoresis for $90 \mathrm{~min}$ with $70 \mathrm{mM}$ Tris- $\mathrm{HCl}$ buffer $(\mathrm{pH} 7.4)$. T+PAl.P. PL, PN or 4-PA: incubated mixture. \#: the drugs were separately spotted at the origin. T: tobramycin. O.: origin.

Figure 3D shows that no significant differences in the TOB binding to the membranes were observed between PALP-treated and non-treated BBMs.

We also studied the cause of the increase in TOB binding to BBMs observed in the case of pretreatment with $0.8 \mathrm{mM}$ of PALP. As shown in Fig. 4A, the pretreatment with $0.6,1.0$ and $1.2 \mathrm{mM}$ of PALP as well as with $0.8 \mathrm{mM}$ of PALP clearly resulted in increases in TOB binding to the membranes, as compared with simultaneous treatment with both compounds. The inhibitory effect of PALP $(0.8$ $\mathrm{mM}$ ) on TOB binding to BBMs disappeared as the preincubation time of PALP and BBMs increased (Fig. 4B). The decrease in the medium osmolarity. which promotes the transport of PALP into BBM vesicles, weakened the inhibitory effect of PALP on TOB binding to BBMs (Fig. 4C).

In addition. PL. PN and 4-PA were ineffective in inhibiting the binding of TOB to BBMs (data not shown).

3. Effect of PALP on intrarenal TOB concentration

As shown in Fig. $5 \mathrm{~A}$, when TOB (90 mg/ $\mathrm{kg} /$ day, s.c.) alone was given to rats, the intrarenal TOB concentrations were $162.6 \pm$ 
A

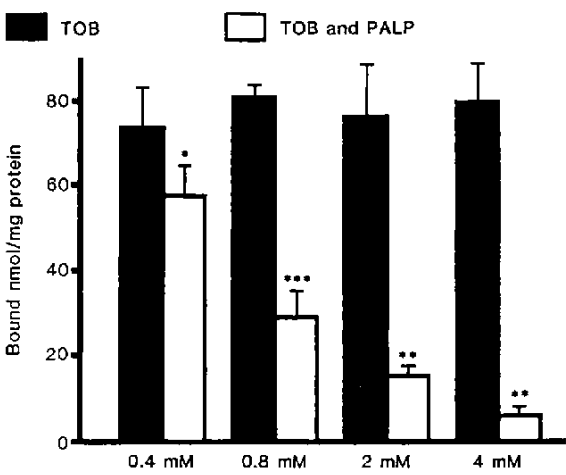

* $: \mathrm{P}<0.05, " *: \mathrm{P}<0.01, \cdots: \mathrm{P}<0.001$, compared to TOB
B

$\square$ TOB and PALP QPZ reaction mixture of TOB and PALP
C

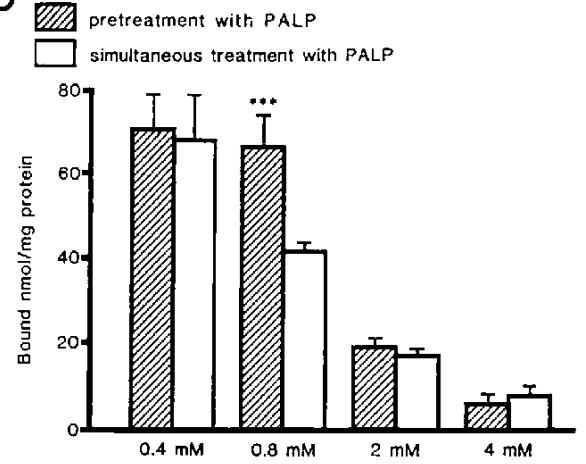

WD pretreatment with PALP

*** : P<0.001, compared to simultaneous treatment with PALP

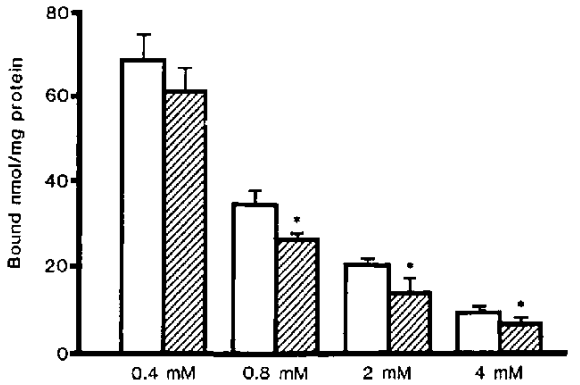

- $\mathrm{P}<0.05$, compared to TOB and PALP

Fig. 3. Effect of simultaneous treatment (A) and pretreatment (C) with PALP, and the reaction mixture of TOB and PALP (B) on TOB binding to BBMs, and the binding of TOB to the PALP-treated BBMs (D). A: PALP $(0.4,0.8 .2$ and $4 \mathrm{mM})$ and TOB $(0.2 \mathrm{mM})$ were added simultaneously to the $B B M$ fraction and incubated for $10 \mathrm{~min}$ at $37^{\circ} \mathrm{C}$. B: TOB and PALP were mixed and preincubated for $3 \mathrm{hr}$ at $37^{\circ} \mathrm{C}$ to form the complex of both drugs. The preincubated reaction mixture was added to the $B B M$ fraction and incubated for $10 \mathrm{~min}$ at $37^{\circ} \mathrm{C}$. C: Pretreatment with PALP: BBMs were preincubated with PALP for 10 min at $37^{\circ} \mathrm{C}$. followed by the incubation with $\mathrm{TOB}$ for $10 \mathrm{~min}$ at $37^{\circ} \mathrm{C}$. Simultaneous treatment with PALP: PALP and TOB were added simultaneously to the BBM fraction and incubated for 10 min at $37^{\circ} \mathrm{C}$. $\mathrm{D}$ : BBMs were preincubated for $10 \mathrm{~min}$ at $37^{\circ} \mathrm{C}$ in a medium (100 mM mannitol, $20 \mathrm{mM} \mathrm{HEPES} / \mathrm{Tris}$. $\mathrm{pH}$ 7.4) with or without PALF, centrifuged at $45,000 \times \mathrm{g}$ for $30 \mathrm{~min}$, and then the membrane pellets were resuspended in the fresh medium (100 $\mathrm{mM}$ mannitol, $20 \mathrm{mM} \mathrm{HEPES/Tris,} \mathrm{pH} 7.4$ ). The resuspended BBMs were incubated for $10 \mathrm{~min}$ at $37^{\circ} \mathrm{C}$ with $0.2 \mathrm{mM}$ of TOB. The values are expressed as the mean \pm S.D. Concentrations shown are the final concentrations.

$30.8 \mu \mathrm{g} / \mathrm{g}$ tissue wet weight and $290.7 \pm$ $51.4 \mu \mathrm{g} / \mathrm{g}$ tissue wet weight on the 1 st and 3 rd days, respectively. The combination of TOB and PALP (200 mg $/ \mathrm{kg} /$ day, p.o.) signif cantly suppressed the TOB level in the kidney $(93.7 \pm 16.0$ and $228.1 \pm 37.6 \mathrm{~kg} / \mathrm{g}$ tissue wet weight on the 1st and 3rd days, respectively)

In addition, each combination of TOB $(70-150 \mathrm{mg} / \mathrm{kg}$. s.c.) and PALP (156-333 $\mathrm{mg} / \mathrm{kg}, \mathrm{p.o}$ ), in which the molar ratios (TOB: PALP $=1: 4$ ) of TOB to PALP were the same, resulted in almost the same inhibitory rate (about $34 \%$ ) on the intrarenal concentration of TOB (Fig. 5B). Figure $5 \mathrm{C}$ shows the effect on the renal TOB level of PALP administered at a different time from that of the TOB injection. The treatment with PA.LP simultaneously and 1 hr before the TOB injection 

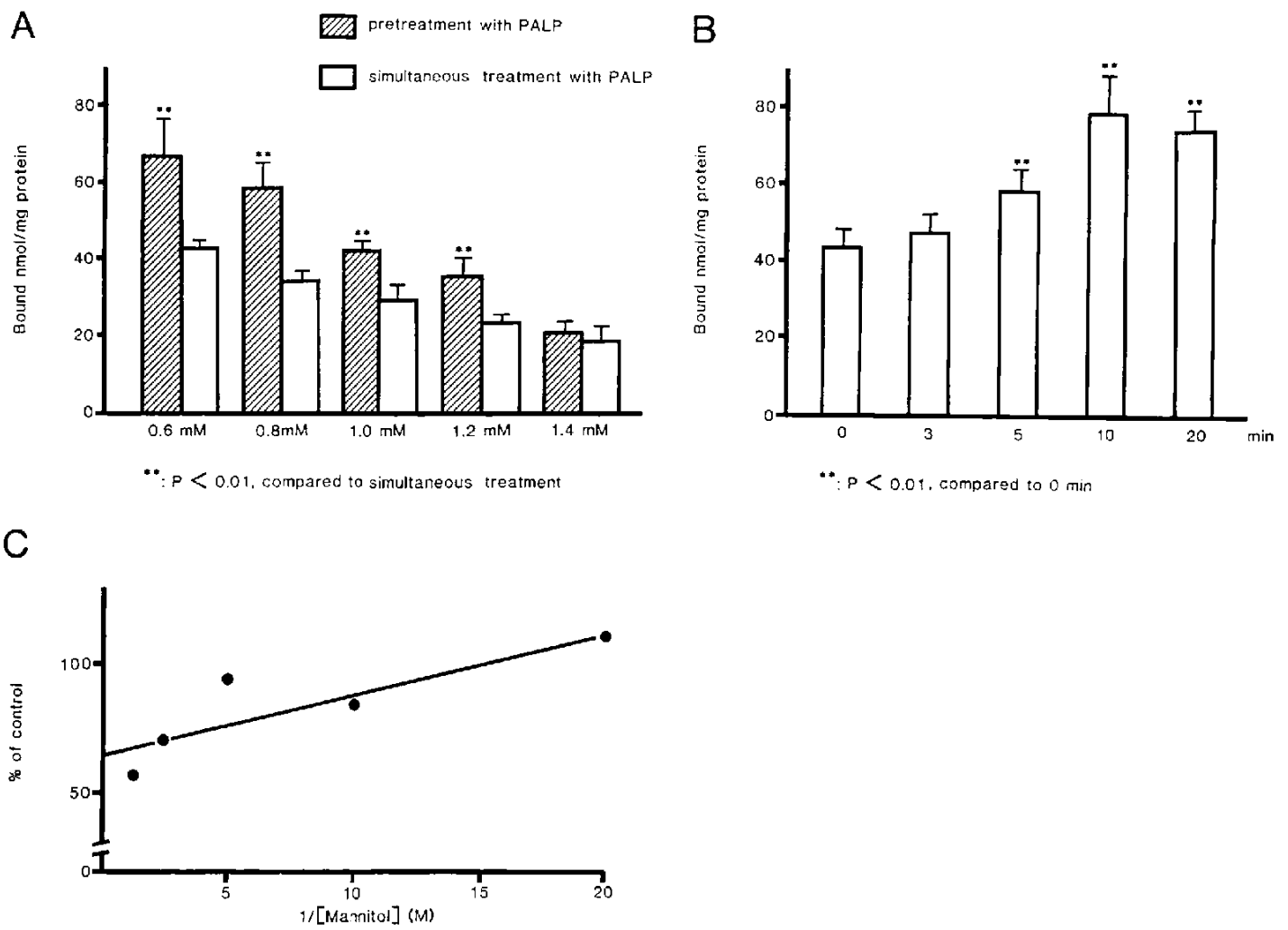

Fig. 4. Effect of pretreatment $(A)$ and preincubation time $(B)$ of PAL.P on TOB binding to BBMs, and the effect of medium osmolarity $(C)$ on the inhibitory effect by PALP or the TOB binding to BBMs. A: Assays were carried out by the method described in Fig. 3C. B: PALP $(0.8 \mathrm{mM})$ was preincubated with BBMs for $3,5,10$ or $20 \mathrm{~min}$ at $37^{\circ} \mathrm{C}$. At a suitable time. TOB $(0.2 \mathrm{mM})$ was added and incubated for $10 \mathrm{~min}$ at $37^{\circ} \mathrm{C}$. C: PALP $(0.8 \mathrm{mM})$ was preincubated with BBMs suspended in the medium with different osmolarity $\left(50,100,200,400\right.$ and $800 \mathrm{mM}$ of mannitol). for $10 \mathrm{~min}$ at $37^{\circ} \mathrm{C}$. followed by the incubation with TOB $(0.2 \mathrm{mM})$ for $10 \mathrm{~min}$ at $37^{\circ} \mathrm{C}$. Simultaneous addition of TOB and PALP to BBMs fraction was performed as a control.

resulted in significant suppression of the TOB level in the kidney. whereas no significant differences in the intrarenal TOB concentration were observed in the treatment with PAL.P at $5 \mathrm{hr}$ before, $5 \mathrm{hr}$ after, or $1 \mathrm{hr}$ after TOB injection.

\section{In vivo nephrotoxicity study}

1) Effect of TOB alone and in combination with PALP on biochemical parameters in urine and blood: Figure 6 illustrates the effect of TOB alone and in combination with PALP on urinary protein content. NAG activity, and BUN content. TOB alone increased urinary protein content and NAG activity, which peaked on the 7 th day and returned to nearly normal levels by the 15th day. On the other hand, combination of TOB and PALP significantly suppressed the increases in urinary protein content and NAG activitv induced by TOB alone by about $90 \%$. Likewise, on the 15 th day, the BUN content in rats given TOB alone was higher by about $80 \%$ than the value in the normal group, whereas the BUN content increased by TOB was markedly suppressed by combination with PALP.

2) Histological observations: On the 7 th day, the kidney from rats receiving TOB alone showed severe tubular cell necrosis and exposure of tubular basement membrane (Fig. 7A). However, as shown in Fig. $7 \mathrm{~B}$ and $\mathrm{C}$, the combination with PALP markedly reduced the degree of cell necrosis induced by TOB. 
A

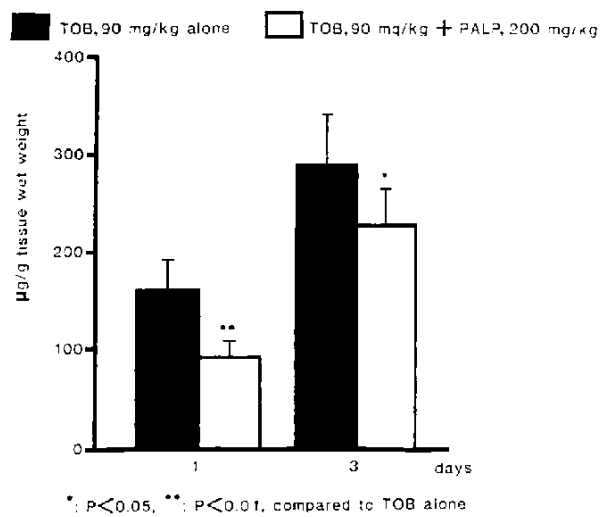

B

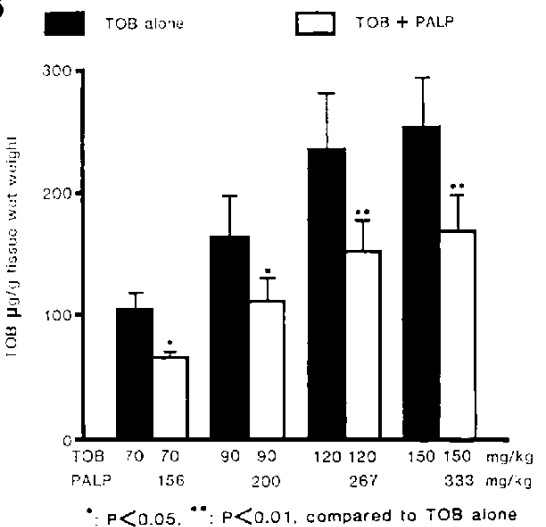

C

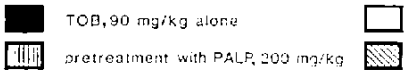

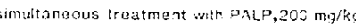
oositreatment wih PALP, .० ming

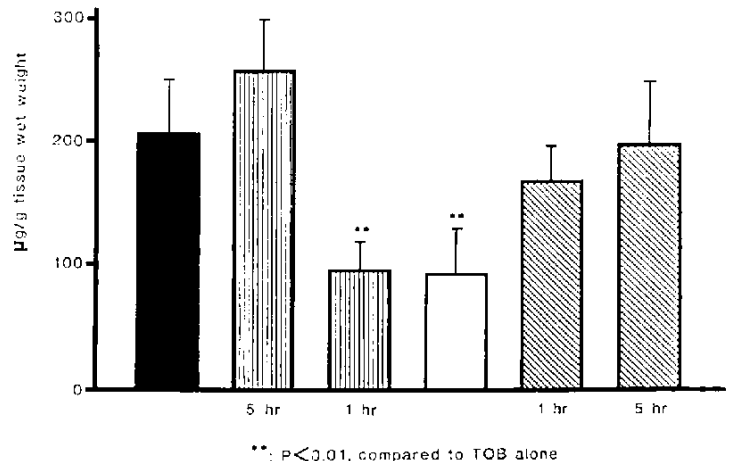

Fig. 5. Effect of PALP on intrarenal concentration of TOB. A: TOB $(90 \mathrm{mg} / \mathrm{kg} /$ day, s.c. $)$ dlone or TOB and PALP (200 mg/kg/day, P.O., $1 \mathrm{hr}$ before TOB injection) were given to rats for 3 days. B: TOB (70$150 \mathrm{mg} / \mathrm{kg}$, s.c.) alone or TOB and PALP (1.56-333 mg/kg, p.o., $1 \mathrm{hr}$ before TOB injection) were given to rats. C: Rats received an s.c. dose of TOB $(90 \mathrm{mg} / \mathrm{kg})$ alone. Other rats groups were given PALP (200 $\mathrm{mg} / \mathrm{kg}$. p.o.) simultaneously with TOB ( $90 \mathrm{mg} / \mathrm{kg}$. s.c.), and before and after ( 1 and $5 \mathrm{hr}$ ) TOB injection.

\section{Discussion}

The purpose of the present study was to examine the ability of PALP to prevent TOBinduced nephrotoxicity.

In the paper electrophoretic analysis, we demonstrated that PALP directly interacted with TOB in vitro, and this was in agreement with the results in our previous study using LMOX (3).

To obtain information about the mechanism of the molecular interaction between TOB and PALP, we examined whether PL, PN and 4$P A$ are able to interact with TOB in vitro. The inability of PN to interact with TOB suggested that phosphate and aldehyde groups in PALP play a role in the molecular interaction of PALP with TOB. The paper electrophoretic analysis using $P L$ suggests that the aldehyde group of PALP. at least in part, contributes to the interaction between PALP and TOB. Keniston (8) reported that polyamines interacted with PALP, and the interaction was due to the formation of a Schiff base between the amino groups of the polyamine and the aldehyde group of PALP. Thus, a Schiff base formed from the amino group of TOB and the aldehyde group of PALP may be involved in the molecular interaction between TOB and PALP. Likewise, PL also may interact with TOB through the formation of a Schiff base. because at $37^{\circ} \mathrm{C}$. PL could induce an elimi- 

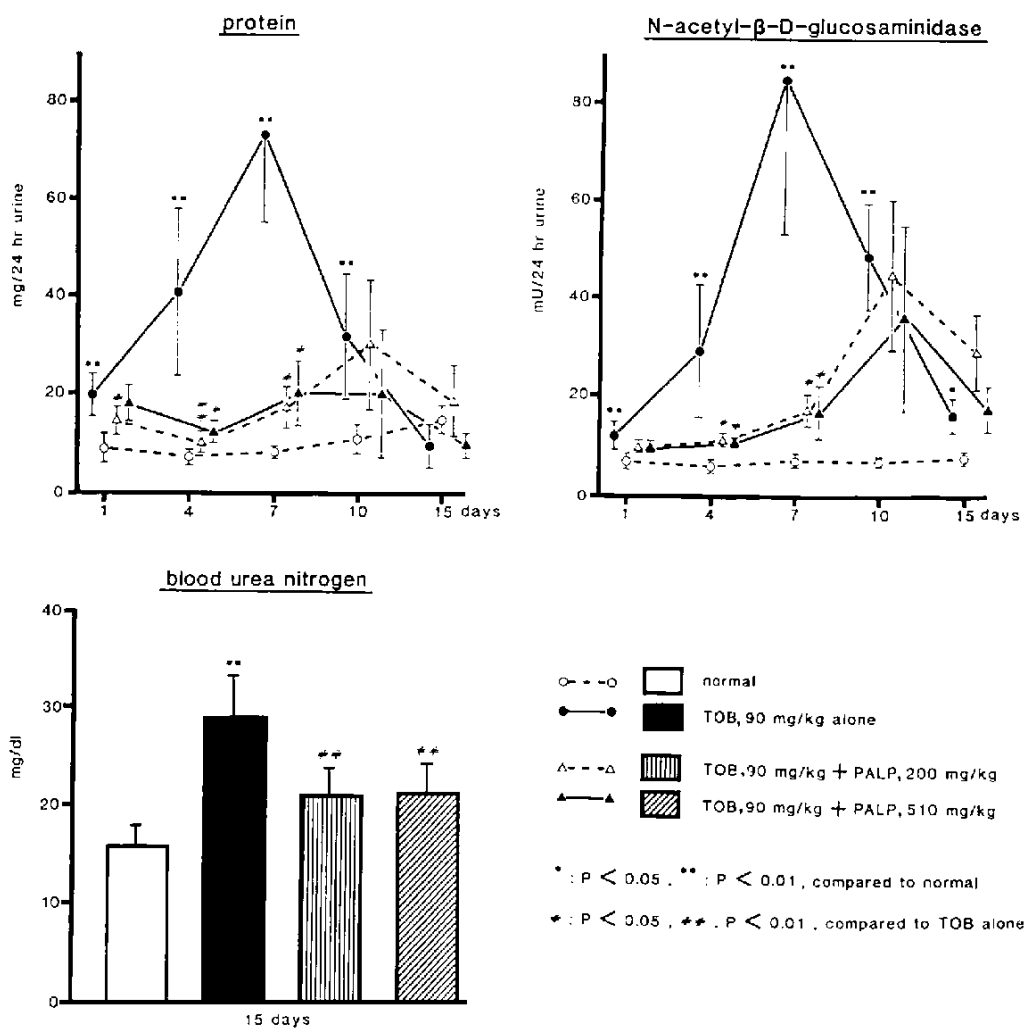

Fig. 6. Effect of TOB alone and in combination with PALP on urinary protein content and $\mathrm{N}$-acetyl- $\beta$ D-glucosaminidase activity and blood urea nitrogen. Each point represents the mean \pm S.D. of 7 rats.
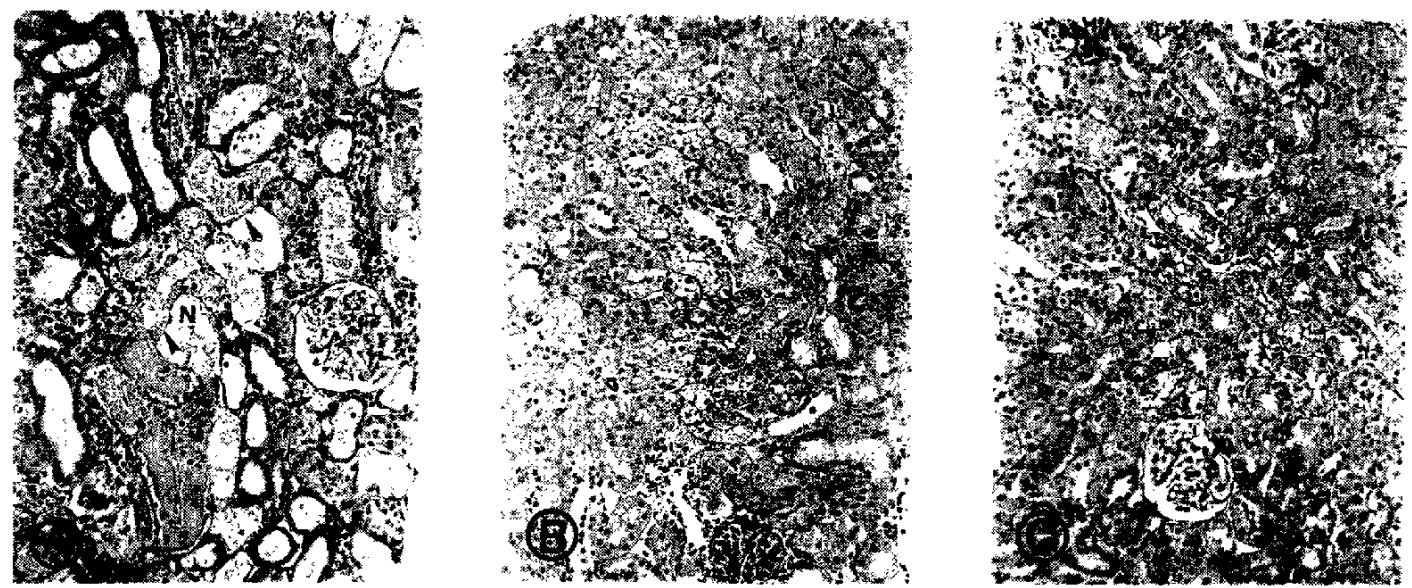

Fig. 7. Light micrograph of rat kidney on the 7th day (PAS stain). A: TOB alone-note the extended necrosis of proximal tubular epithelıal cells $(N)$ and residual tubular basement membrane (arrow head). B: TOB +PALP. $200 \mathrm{mg} / \mathrm{kg}$ : C: TOB-PALP. $510 \mathrm{mg} / \mathrm{kg}$-the degree of necrosis of tubular cells was reduced. 
nation reaction of serine 3-phosphate to inorganic phosphate, pyruvate and ammonia, which was mediated by the formation of a Schiff base between the amino group of serine 3 -phosphate and the aldehyde group of PL (9).

Additionally, since the degree of disappearance of the spot corresponding to $T O B$ in the mixture of TOB and PALP seemed to be greater than that in the TOB-PL mixture, it is very likely that the negatively charged phosphate group in PALP also would be involved in the interaction with TOB through, probably. the ionic binding to the positive charge of the protonated amino group of TOB at a very early stage of the interaction.

In the assay of TOB binding to BBMs, PALP added simultaneously with TOB to the membrane fraction significantly inhibited the binding of TOB to BBMs (Fig. 3A). The lower amount of TOB binding to BBMs observed by the addition of the TOB-PALP mixture suggested that the inhibitory action of PALP on TOB binding to BBMs may be attributed to the molecular interaction be tween TOB and PALP (Fig. 3B). Moreover. the experiments concerning pretreatment with PALP (except for $0.8 \mathrm{mM}$ of PALP) (Fig. $3 B$ ) and TOB binding to the PALP-treated BBMs (Fig. 3D) indicated that even if PALP bound to BBMs, it would not be associated with the inhibition by PAL.P of the binding of TOB to the membranes.

Additionally, it was evident that the decrease in the inhibitory action of PALP on TOB binding to BBMs was induced by preincubation of PALP and BBMs not only in the case of the $0.8 \mathrm{mM}$ of PALP but also at other concentrations of PALP (Fig. 4A). The decrease in the capability of PALP to inhibit the TOB binding to BBMs, which was observed in Fig. $4 B$ and $C$, suggests a contribution of PALP existing outside, but not inside, of the $B B M$ vesicles to the inhibition of TOB binding to BBMs.

Moreover, the inability of $\mathrm{PL}$ to suppress the TOB binding to BBMs suggests the importance of the negatively charged phosphate group of PALP in inhibiting the binding of TOB to the membranes. The evidence that PN and 4-PA, which did not show an obvious interaction with TOB. were ineffective in in- hibiting the TOB binding to BBMs also may imply a possible involvement of the interaction between PALP and TOB in the inhibition by PALP of the binding of TOB to BBMs. In addition, we confirmed by thin layer chromatography the interaction of TOB with PALP which occurred during the incubation process (data not shown). Thus, the results of the binding assay indicate that PALP inhibits the binding of TOB to BBMs and that the inhibitory action of PALP is due to the interaction of PALP and TOB outside of BBM vesicles.

in our study, we found that PALP treatment significantly inhibited the intrarenal TOB concentration (Fig. 5A). This result was in agreement with the observation reported by Kacew (10), using gentamicin and PALP. It has been shown that spermine, a polyamine, reduced the gentamicin uptake in the rat kidney cortex (11) and that the binding of gentamicin to rat BBMs was inhibited by spermine (12). These observations suggested that reduction of intrarenal gentamicin level was associated with the inhibition of gentamicin binding to BBMs. Thus, the suppression by PALP of the intrarenal TOB level may result from the inhibition of TOB binding to BBMs by PALP.

Moreover, we found the similar inhibitory rate of intrarenal TOB concentration by PALP in the each combination of TOB and PALP (Fig. 5B) and the limited administration time of PALP to suppress the renal accumulation of TOB (Fig. 5C). These observations suggest that the reduced $T O B$ level in the kidney may be due to the interaction of both compounds which leads to less binding of TOB to BBMs. rather than due to the physiological action of PALP in the kidney. The existence of a complex of polyamine and PALP in urine (13) may strengthen the above possibility, although in this study, the detection of a TOBPALP complex in vivo was not attempted.

Finally, in the in vivo nephrotoxicity study, we demonstrated that PALP prevented the development of nephrotoxicity induced by TOB, as elucidated by the biochemical (Fig. 6) and histological (Fig. 7) observations.

Because it has been suggested that the level of aminoglycoside antibiotics (AGs) in the kidney is related to the degiee of the nephrotoxicity of AGs (14) (and indeed, we also confirmed a positive correlation between 
the suppression rate of the intrarenal TOB level by PALP and the degree of suppression by PAL.P of BUN and plasma creatinine levels elevated by TOB (data not shown)), the protective action of PALP against TOB-induced nephrotoxicity may be due to reduction of the intrarenal concentration of TOB by PALP.

However, recently, it has been suggested that the binding of AGs with biomembranes and liposomal membranes, which leads to alterations in several membrane functions (15-19), plays an important role in the pathogenesis of AGs-induced nephrotoxicity. Williams et al. (20) have proposed the significance of the binding affinity of AGs to BBMs in the toxic action of $A G$ s on rat kidneys. Thus, our finding that PALP inhibited the binding of TOB to BBMs also may be included in the explanation of the mechanism of the protective action of PALP against TOB nephrotoxicity.

In conclusion, the results of the present study indicate that PALP obviously protects rat kidneys from nephrotoxicity induced by TOB and that the protective action of PALP against the nephrotoxicity of TOB may be due to the reduction in intrarenal TOB concentration and the inhibition of the binding of TOB to BBMs. Additionally, the interaction of TOB and PALP may, at least in part, play a role in the protective effect of PALP against TOB nephrotoxicity.

Acknowledgement: We thank Shionogi Co., Ltd. for supplying TOB.

\section{References}

1 Kojima, R., Ito, M. and Suzuki, Y.: Studies on the nephrotoxicity of aminoglycoside antibiotics and protection from these effects (3). Protective effect of latamoxef against tobramycin nephrotoxicity and its protective mechanism. Japan. J. Pharmacol. 42, 397-404 (1986)

2 Kojima, R., Ito, M. and Suzuki, Y.: Studies on the nephrotoxicity of aminoglycoside antibiotics and protection from these effects (7): Effect of latamoxef on binding of tobramycin to brush border membranes isolated from rat kidney cortex. Japan. J. Pharmacol. 51, 465-473 (1989)

3 Kojima, R., Ito, M. and Suzuki, Y.: Studies on the nephrotoxicity of aminoglycoside antibiotics and protection from these effects (5). Interaction of tobramycin with latamoxef in vitro. J. Pharmacobiodyn. 11, 9-17 (1988)
4 Evers, C., Haase, W., Murer, $H$. and Kinne, R.: Properties of brush border vesicles isolated from rat kidney cortex by calcium precipitation. Membr. Biochem. 1, 203-219 (1978)

5 Inui, K., Okano, T., Takano, M., Kitazawa, S. and Hori, R.: A simple method for the isolation of basolateral plasma membrane vesicles from rat kidney cortex: Enzyme activities and some properties of glucose transport. Biochim. Biophys. Acta 647, 150-154 (1981)

6 Ishikawa, Y., Inui, K. and Hori, R.: Gentamicin binding to brush border and basolateral membranes isolated from rat kidney cortex. J. Pharmacobiodyn. 8, 931-941 (1985)

7 Burd, F.J., Wong, C.R., Feerey, E.J., Carrico, J.R. and Boguslaski, C.R.: Homogeneous reactantlabeled fluorescent immunoassay for therapeutic drugs exemplified by gentamicin determination in human serum. Clin. Chem. 23, 1402-1408 (1977)

8 Keniston, C.R.: Polyamine-pyridoxal 5'-phosphate interaction: Effects of $\mathrm{pH}$ and phosphate concentration in Schiff's base formation. Physiol. Chem. Phys. 11, 465-470 (1979)

9 Longenecker, B.J. and Snell, E.E.: The comparative activities of metal ions in promoting pyridoxal-catalyzed reactions of amino acids. J. Am. Chem. Soc. 79, 142-145 (1957)

10 Kacew, S.: Inhibition of gentamicin-induced nephrotoxicity by pyridoxal-5'-phosphate in the rat. J. Pharmacol. Exp. Ther. 248, 360-366 (1989)

11 Josepovits, C., Pastoriza-Munoz, E., Timmerman, D., Scott, M., Feldman, S. and Kaloyanides, J.G.: Inhibition of gentamicin uptake in rat renal cortex in vivo by aminoglycosides and organic polycations. J. Pharmacol. Exp. Ther. 223, 314 321 (1982)

12 Williams, D.P., Hottendorf, H.G. and Bennett, B.D.: Inhibition of renal membrane binding and nephrotoxicity of aminoglycosides. J. Pharmacol. Exp. Ther. 237, 919-925 (1986)

13 Aigner-Held, R., Campbell, A.R. and Daves, D.G., Jr.: Polvamine-pyridoxal Schiff bases in urine. Proc. Natl. Acad. Sci. U.S.A. 76, 6652-6655 (1979)

14 Ramsammy, S.L., Josepovitz, C., Jones, D., Ling, K.Y. Lane, B.P. and Kaloyanides, G.J.: Induction of nephrotoxicity by high doses of gentamicin. Proc. Soc. Exp. Biol. Med. 186, 306312 (1987)

15 Marche, P., Koutouzov, S. and Girard, A.: Impairment of membrane phosphoinositide metabolism by aminoglycoside antibiotics: Streptomycin, amikacin, kanamycin, dibekacin, genta- 
micin and neomycin. J. Pharmacol. Exp. Ther. 227, 415-420 (1983)

16 Chung, L., Kaloyanides, J.G., McDaniel, R., McLaughlin, A. and McLaughlin, S.: Interaction of gentamicin and spermine with bilayer membrane containing negatively charged phospholipids. Biochemistry 24, 442-452 (1985)

17 Horio, M., Fukuhara, Y., Orita, Y., Nakanishi, T., Nakahama, H., Moriyama, T. and Kamada, T.: Gentamicin inhibits $\mathrm{Na}^{+}$-dependent $\mathrm{D}$-glucose transport in rabbit kidney brush-border membrane vesicles. Biochim. Biophys. Acta 858, 153-160 (1986)

$18 \mathrm{Au}$, S., Schacht, J. and Weiner, N.: Membrane effects of aminoglycoside antibiotics measured in liposomes containing the fluorescent probe. 1 -anilino-8-naphthalene sulfonate. Biochim. Biophys. Acta 862, 205-210 (1986)

$19 \mathrm{Au}$, S., Weiner, D.N. and Schacht, J.: Aminoglycoside antibiotics preferential increase permeability in phosphoinositide-containing membranes: A study with carboxyfluorescein in liposomes. Biochim. Biophys. Acta 902, 80-86 (1987)

20 Williams, P.D., Bennett, D.B., Gleason, C.R. and Hottendorf, G.H.: Correlation between renal membrane binding and nephrotoxicity of aminoglycosides. Antimicrob. Agents Chemother. 31. $570-574(1987)$ 